\title{
Formation and Evolution of Black Hole X-ray Transient Systems
}

\author{
G.E. Brown ${ }^{\mathrm{a}, 1}$ C.-H. Lee ${ }^{\mathrm{a}, \mathrm{b}, 2}$ T.M. Tauris ${ }^{\mathrm{c}, 3}$ \\ ${ }^{a}$ Department of Physics $\&$ Astronomy, State University of New York, \\ Stony Brook, New York 11794, USA \\ ${ }^{\mathrm{b}}$ School of Physics, Korea Institute for Advanced Study, Seoul 130-012, Korea \\ ${ }^{\mathrm{c}}$ Nordic Institute for Theoretical Physics (NORDITA), Blegdamsvej 17, DK-2100 \\ Copenhagen Ø, Denmark
}

\begin{abstract}
We study the formation of low-mass black hole X-ray binaries with main sequence companions that have formed through case $\mathrm{C}$ mass transfer (mass transfer following the helium core burning phase of the black hole progenitor). We identify these objects with the observed soft X-ray transients. Although this scenario requires a set of fine tuned conditions, we are able to produce a current Galactic population of $\sim 2000$ objects, in agreement with estimates based on observations.

The narrow interval in initial separations leading to case $\mathrm{C}$ mass transfer, combined with the allowed narrow range of separations after the common envelope evolution, constrains the common envelope efficiency in this scenario: $\lambda \alpha_{c e} \approx 0.2-0.5$.
\end{abstract}

Key words: black hole physics — stars: binaries: close — accretion

1 E-mail: popenoe@nuclear.physics.sunysb.edu

2 E-mail: chlee@kias.re.kr

3 E-mail: tauris@nordita.dk

Preprint submitted to Elsevier Preprint 27 January 2019 


\section{Introduction}

As shown by Brown, Weingartner \& Wijers (1996), massive stars which lose their hydrogen envelope during binary evolution evolve differently from single stars. Particularly, when the high mass-loss rates that are found for Wolf-Rayet stars are applied to the exposed "naked" helium cores formed in binaries, all massive stars up to $60 M_{\odot}$ evolving through case A (Roche-lobe overflow during core hydrogen burning) or case B (RLO before core helium burning) mass transfer will form a rather low-mass $\left(M<4 M_{\odot}\right)$ helium star with a $\lesssim 1.5 M_{\odot}$ Fe core (Wellstein \& Langer 1999; Brown et al. 2000a). These stars therefore explode in a supernova and form a neutron star or a low-mass black hole instead.

Brown, Lee, \& Bethe (1999) suggested that high-mass $\left(\sim 7 M_{\odot}\right)$ black holes can only be formed in binaries when the massive stars lose their hydrogen envelope after core helium burning has ceased (so-called case C mass transfer) and calculated the expected number of systems that can be formed through this scenario. However, they did not follow the evolution of the formed X-ray binaries in detail and also did not take wind losses into account, which has a large influence on the evolution of massive stars in binaries (Portegies Zwart, Verbunt \& Ergma 1997).

In this note we consider the evolution of those low-mass black hole X-ray binaries with main sequence companions which are formed through case $\mathrm{C}$ mass transfer followed by common envelope and spiral-in evolution. We estimate their expected number currently present in our Galaxy. We therefore discuss the following.

(1) The initial separation interval of binaries that go through case $\mathrm{C}$ mass transfer and the ZAMS mass interval of stars being able to produce highmass black holes (Sect. 2).

(2) The initial masses of the black hole companions (the donors in the presentday X-ray binaries) and the allowed orbital periods after the common envelope required for the systems to become observable X-ray binaries within the age of our Galaxy before the donors leave the main-sequence (Sect. 3).

(3) The resulting common envelope efficiency that can be estimated combining points 1 and 2 (Sect. 4).

(4) The evolution of the mass-transfer rate in the X-ray transient phase and the lifetimes of the systems (Sect. 5).

(5) The total number of systems expected in our Galaxy at present (Sect. 6).

(6) The related black hole systems with (sub)giant companions and why they may have formed in a different way (Sect. 7). 


\section{Case C mass transfer: Limits on Initial Separations and Black Hole Progenitor Masses}

In order to have case $\mathrm{C}$ mass transfer the radius of the star has to expand after core helium burning has stopped. In the calculation of Schaller et al.(1992), stars with ZAMS masses $20 M_{\odot}$ and $25 M_{\odot}$ satisfy the requirement. However, during the core helium burning stage, the orbit expands due to the mass loss:

$$
\frac{a_{\Delta M}^{\prime}}{a}=\frac{M+M_{d}}{M-\Delta M+M_{d}}
$$

where $a$ is the orbital separations, $M$ is the mass of the black hole progenitor, $\Delta M$ is the mass lost from the black hole progenitor during the core helium burning, and $M_{d}$ is the donor star mass. In order to initiate mass transfer after core helium burning the star has to expand sufficiently that this widening of the orbit is compensated for.

In the upper curve of Fig. 1 we draw the orbital separation of the binary required to initiate Roche lobe overflow calculated at each stage from the radii given by Schaller et al. (1992). For the masses considered the radius of giant star is at $\sim 2 / 3$ of the radial separation between the two stars. The solid curve gives the corresponding initial separation between the massive star and the low-mass main sequence companion after strong wind mass loss is switched on. Here we assume that the wind mass loss does not affect the stellar radius. 4 We see that in the case of a $20 M_{\odot}$ star the mass transfer can start following He core burning for binaries with an initial separation in the interval of $1370 R_{\odot}<a_{\mathrm{i}}<1605 R_{\odot}$. In the case of a $25 M_{\odot}$ star, however, mass transfer is only possible up to just after the beginning of core helium burning (with initial separations around $1170 R_{\odot}$ ). Binaries with larger initial separations will become too wide during core helium burning so even though the star expands slightly after core helium burning, it will not be able to fills its Roche lobe (see also Fig. 2 of Portegies Zwart, Verbunt, \& Ergma 1997).

Brown, Weingartner \& Wijers (1996) found that stars with ZAMS masses above $19 M_{\odot}$, evolved by Woosley \& Weaver (1995), evolve into high-mass $\left(\sim 7 M_{\odot}\right)$ black holes. As outlined in Brown et al. (2000a), this requires the special rate of $170 \mathrm{keV}$ barns at energy $E=300 \mathrm{keV}$ for the ${ }^{12} \mathrm{C}(\alpha, \gamma){ }^{16} \mathrm{O}$ reaction used by Woosley \& Weaver. A ZAMS $18 M_{\odot}$ star, which is usually taken as progenitor for SN 1987A, will form a low-mass $\left(\sim 1.5-1.8 M_{\odot}\right)$ black

$\overline{4}$ One of us (T.M.T.) has carried out stellar evolution calculations, using Eggleton's code, with/without the wind mass losses, and find that the radius behaves almost the same. The minor difference will give rise to adjusting our numbers slightly, but the outline will remain the same. 

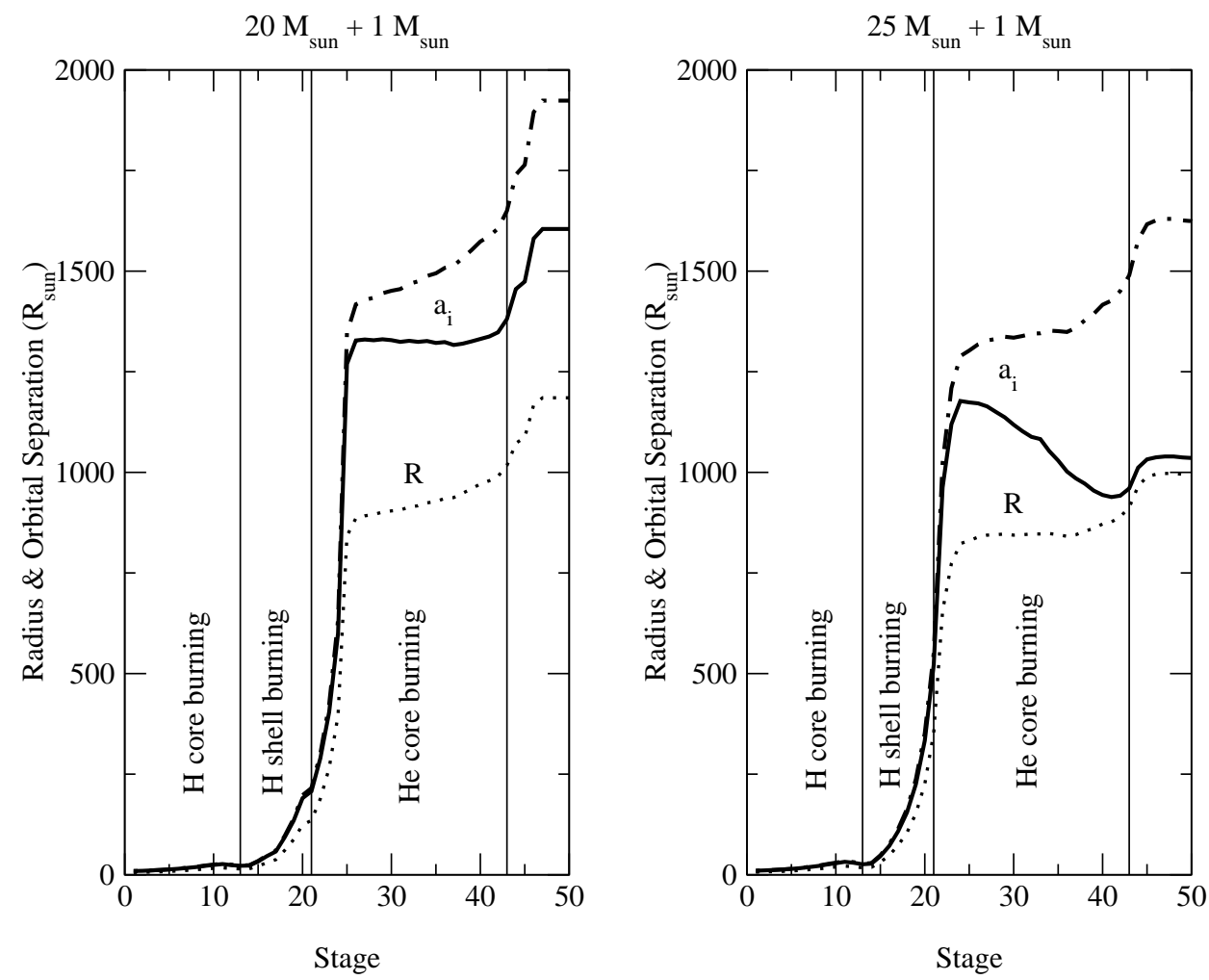

Fig. 1. Radius of black hole progenitors $(R)$ and the initial orbital separations $\left(a_{i}\right)$ of the progenitors of X-ray transient binaries with a $1 M_{\odot}$ companion. Upper dot dashed curves correspond to the orbital separations required to initiate Roche lobe overflow at the radius taken for each stage from Schaller et al. (1992). The solid curves correspond to the required initial separations after corrections of the orbit widening due to the wind mass loss. The lower dotted curves correspond to the radius of the black hole progenitors taken from Schaller et al. (1992).

hole or a neutron star.

Hence, the mass interval of stars that can go through case $\mathrm{C}$ mass transfer and collapse into a high-mass black hole is limited between 19 and 20-25 $M_{\odot}$. We shall (arbitrarily) take $22 M_{\odot}$ as an upper limit. Stellar evolution calculations with lower wind mass losses might give a higher mass limit.

Using the above derived limits on the mass of the black hole progenitors and the initial separation, the rate of formation of black hole transient sources with main sequence companions we can evolve (see Section 6) is nearly an order of magnitude less than in Brown, Lee \& Bethe (1999) where wind mass losses were ignored. We realize that our evolution requires a fine-tuned parameter space, but we shall argue that it all hangs together. The exact numbers may alter with changes in stellar evolution models. 


\section{Limits on Donor Masses and Orbital Periods}

During the common envelope evolution the low-mass main sequence star of mass $\sim 1 M_{\odot}$ will spiral into the envelope of the massive giant. The orbit will shrink dramatically and the outcome is a close binary consisting of the core of the massive giant and the low-mass star (Paczyński 1976). Shortly after the spiral-in and ejection of the envelope of the giant, the remaining core will collapse into a black hole. Whether or not this collapse is associated with mass loss is still an open question. The orbit will then shrink further due to the loss of orbital angular momentum via magnetic braking and gravitational wave radiation (e.g. Verbunt 1990) until the low-mass (donor) star begins to transfer mass to the black hole - forming an X-ray binary. Since the wind mass-loss rate of the low-mass donor star is very low, and its accretion during the short common envelope phase is negligible, it will have a mass at the onset of the X-ray phase which is about equal to its initial mass.

The time evolution of the orbital separation is described as

$$
\frac{\dot{a}}{a}=\frac{2 \dot{J}_{g w}}{J_{o r b}}+\frac{2 \dot{J}_{m b}}{J_{o r b}}-2 \dot{M}_{d}\left(\frac{M_{B H}-M_{d}}{M_{B H} M_{d}}\right) .
$$

Here we assume that the mass lost from the companion star is all accreted onto the black hole. The orbital angular momentum loss by gravitational wave radiation ( $\mathrm{gw}$ ) and magnetic braking $(\mathrm{mb})$ is given by:

$$
\begin{aligned}
& \frac{\dot{J}_{g w}}{J_{\text {orb }}}=-\frac{32 G^{3}}{5 c^{5}} \frac{M_{B H} M_{d}\left(M_{B H}+M_{d}\right)}{a^{4}} \mathrm{~s}^{-1} \\
& \frac{\dot{J}_{m b}}{J_{\text {orb }}} \approx-0.5 \times 10^{-28} b_{m b} \frac{I R_{d}^{2}}{a^{5}} \frac{G\left(M_{B H}+M_{d}\right)^{2}}{M_{B H} M_{d}} \mathrm{~s}^{-1}
\end{aligned}
$$

where $R_{d}$ is the radius of the donor star, $b_{m b}$ is an efficiency parameter given below, and $I \approx 0.1 M_{d} R_{d}^{2}$ is the moment of inertia of the donor star. Here we have assumed a magnetic braking law based on observations of slowly rotating single stars by Skumanich (1972). (However, this law may be too strong, i.e. overestimating the dependence on the angular velocity, see e.g. Stepien 1995). We further assumed the the magnetic braking efficiency suggested by Kalogera \& Webbink (1998),

$$
b_{m b}\left(M_{d}\right)=\left\{\begin{array}{lr}
0, & M_{d} \leq 0.37 M_{\odot} \\
1, & 0.37 M_{\odot}<M_{d}<1.03 M_{\odot} . \\
\exp \left(-4.15\left(M_{d}-1.03\right)\right), & M_{d}>1.03 M_{\odot}
\end{array}\right.
$$




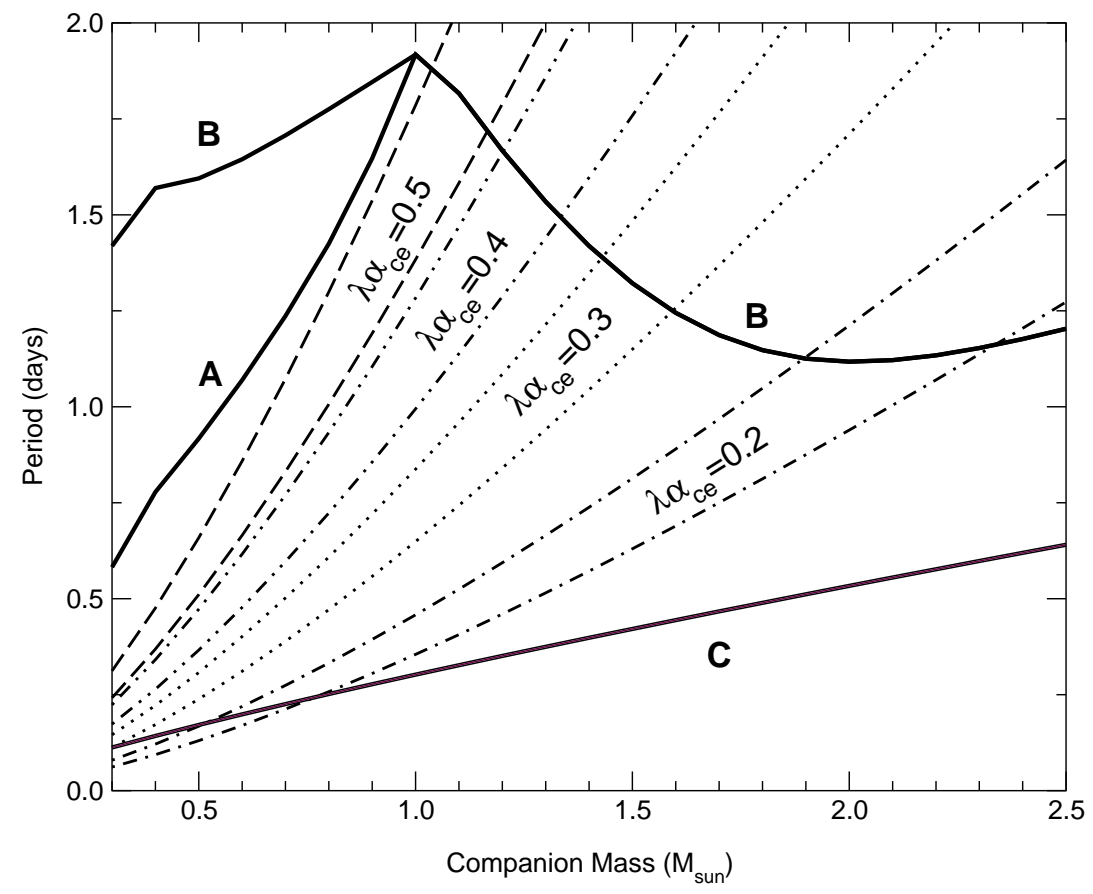

Fig. 2. Limits on the orbital period after the formation of the black hole as function of the mass of the low-mass companion. The upper and lower boundaries (thick solid lines) of mass and period are set by the conditions (see Section 3): A) Mass transfer begins within the Hubble time, B) Mass transfer begins before the companion evolves off the main sequence, C) Mass transfer from the companion begins right after finishing common envelope evolution. The expected ranges of periods and companion masses, after the common envelope phase, are plotted for various given efficiencies (see Section 4). The width of each band for an assumed $\lambda \alpha_{c e}$ is determined by the initial band of possible Roche Lobe overflows with Case $\mathrm{C}$ mass transfer. We took the ZAMS mass of the black hole progenitor to be $20 M_{\odot}$ which corresponds to $M_{p} \sim 16.8 M_{\odot}$ in the beginning of case $\mathrm{C}$ mass transfer, and $M_{H e}=M_{B H}=7 M_{\odot}$.

Here the effect of magnetic braking is assumed to be strongest for stars with a mass $\approx 1.0 M_{\odot}$. Donor stars with mass $>1.5 M_{\odot}$ do not have a convective envelope on the main sequence and the magnetic braking effects are therefore negligible, so the binary evolution is dominated by gravitational radiation until the mass transfer starts.

In Fig. 2, we show the possible ranges of orbital periods and donor masses immediately after the formation of the black hole, such that an X-ray binary with a main-sequence donor is formed within the Hubble time (Kalogera 1999). If no mass is lost in the formation of the black hole, these periods are equal to the periods immediately after the common envelope as a result of the short time interval before the collapse of the core.

The upper limit is set by two conditions: i) the binary should start mass transfer within the Hubble time (line denoted by A), ii) the mass transfer 
should start before the donor evolves off the main-sequence (line denoted by B) which also determines the lower limit on $\lambda \alpha_{c e}=0.2$ (see Section 4 ). The kink in line $\mathrm{B}$ around $\sim 1 M_{\odot}$ comes from the fact that the effect of magnetic braking, eq.(4), is strongest for stars with a mass $\sim 1 M_{\odot}$. For this plot, we took the simple interpolation of Schaller's results for the evolution of a $1 M_{\odot}$ star with two extrapolations for the radius $R$ and the lifetimes of the mainsequence star $t_{\text {end ms; }}$

$$
\begin{aligned}
R\left(M_{d}, t\right) & =R_{M_{\odot}}(t) \times M_{d}^{0.88} \\
t_{\text {end } \mathrm{ms}}\left(M_{d}\right) & = \begin{cases}t_{M_{\odot}} / M_{d}^{2} & \text { for } M_{d}<1 M_{\odot} \\
t_{M_{\odot}} / M_{d}^{2.5} & \text { for } M_{d}>1 M_{\odot}\end{cases}
\end{aligned}
$$

where $t_{M_{\odot}}$ is the time at the stage 13 of the $1 M_{\odot}$ model of Schaller et al. 1992 (see Fig. 1). The applied formulae may not be very accurate, but the qualitative behaviour after more realistic numerical calculations will remain the same. The lower boundary of the period immediately after the common envelope (line denoted by $\mathrm{C}$ ) is set by the condition that the main sequence star should not overfill its Roche-lobe at the end of the common envelope.

\section{Common Envelope Evolution: Binding Energy and Efficiency Pa- rameter}

Since our initial separation $a_{\mathrm{i}}$ is sharply defined by the condition of case $\mathrm{C}$ mass transfer, and our final separations are constrained by the distance that can be traversed during magnetic braking and gravitational wave radiation, we can determine the range of allowed common envelope efficiencies in order to form low-mass black hole X-ray transients.

During the common envelope phase the energy needed to expel the hydrogen envelope of the black hole progenitor is tapped from the drop in binary orbital potential energy with efficiency $\alpha_{\text {ce }}$ (also denoted $\eta$ in the literature):

$$
\frac{G M_{p} M_{e}}{\lambda R}=\frac{G M_{p} M_{e}}{\lambda r_{L} a_{i}}=\alpha_{c e}\left(\frac{G M_{H e} M_{d}}{2 a_{f}}-\frac{G M_{p} M_{d}}{2 a_{i}}\right)
$$

where $M_{p}$ is the total mass of the $\mathrm{BH}$ progenitor star just before the common envelope forms, $M_{e}$ is the mass of its hydrogen envelope, $M_{H e}$ is the mass of its core, $a_{\mathrm{i}}$ and $a_{\mathrm{f}}$ is the initial and final separation, before and after the common envelope, respectively. $r_{L}$ is the dimensionless Roche-lobe radius. Given the parameters of the system at the start of the common envelope, the final separation is determined by $\lambda$, describing the structure of the giant 
and $\alpha_{c e}$, the efficiency of the energy conversion. In our case the final separation is limited as shown in Fig. 2 so the product $\lambda \alpha_{c e}$ can be constrained. In the literature $\lambda=0.5$ had often been used, and a high efficiency $\alpha_{c e}>1$ was often required in order to explain the observations. However, recent detailed stellar evolution calculations by Dewi \& Tauris (2000) show that $\lambda$ can be substantially larger. These high values of $\lambda$ solve the problem of unrealistically high efficiencies $\alpha_{c e}$. For a $20 M_{\odot}$ star, corresponding to $16.2 M_{\odot}$ at the tip of the AGB, Tauris \& Dewi (2001) find $\lambda$-values in a large interval: $0.1<\lambda<3$ depending on the exact location of the core mass boundary and amount of internal thermodynamic energy included.

In Fig. 2 we plotted the possible ranges of periods after the common envelope. We took the ZAMS mass of the black hole progenitor to be $20 M_{\odot}$, which corresponds to $M_{p} \sim 16.8 M_{\odot}$ in the beginning of case $\mathrm{C}$ mass transfer, and assumed $M_{H e}=M_{B H}=7 M_{\odot}$. For each value of $\lambda \alpha_{c e}$ the two lines are for the limiting initial separations $a_{\mathrm{i}}$ (see Sect. 2). Donor stars with masses above $1.5 M_{\odot}$ can only be formed if the common envelope efficiency $\lambda \alpha_{c e}$ is around 0.2 .

\section{Life Time of X-ray Transient Sources}

Brown, Lee \& Bethe (1999), assuming a mass-loss rate of $10^{-9} M_{\odot} \mathrm{yr}^{-1}$, obtained a lifetime of the X-ray transients of $10^{9}$ yrs . However, as Ergma \& Fedorova (1998) discussed, the mass-loss rate changes as a function of time.

Once the Roche lobe overflow starts, at any given time, the orbital separation is self determined in order for the companion star to fill its Roche lobe,

$$
a=R_{d} / r_{L}\left(M_{d}, M_{B H}\right)
$$

Hence, the mass loss rate is automatically determined by the feedback effects between the orbital widening due to the mass loss and the orbital contraction due to the gravitational wave radiation and the magnetic braking, eq. (2). In the numerical simulation of mass transfer due to Roche lobe overflow, for a given $\delta M_{d}$, we can get the $\delta t$, equivalently $\dot{M}_{d}$, by requiring the donor star fill its Roche lobe.

In Fig. 3 we plot the evolution of the donor star mass and the mass loss rate as a function of time for $M_{d, i}=1.25 M_{\odot}$ with a $7 M_{\odot}$ black hole. There are two tracks after Roche lobe overflow.

(1) Case TO: we assumed that the stripped star after Roche lobe overflow continues to follow exactly the same evolution time scale as the original 

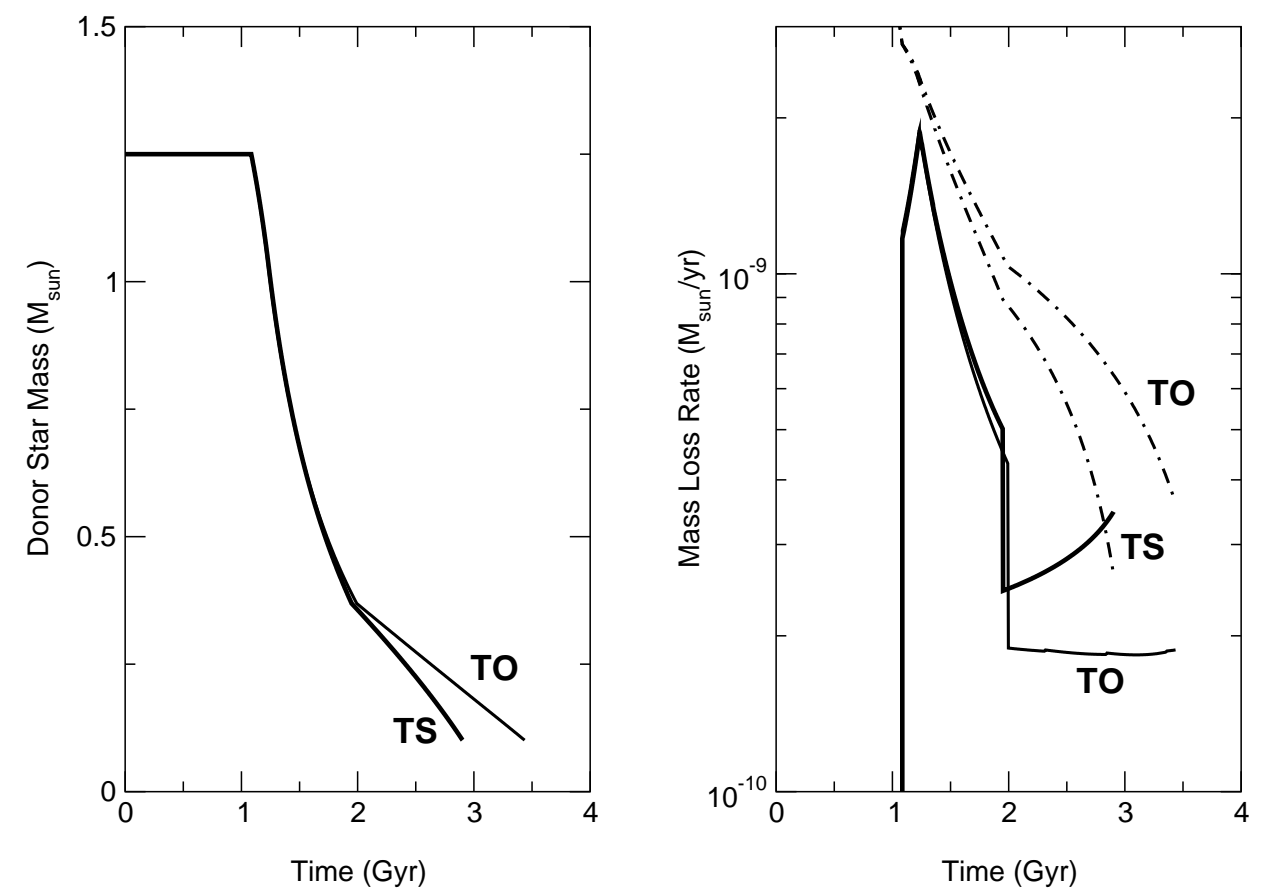

Fig. 3. Time evolution of the donor star mass and mass loss rate for $M_{d, i}=1.25 M_{\odot}$ with a $7 M_{\odot}$ black hole and an initial period of 20 hours. The dot-dashed lines on the right panel correspond to the critical mass loss rate for the steady X-ray sources, eq. (8). The two tracks correspond to the different time scales of stripped donor stars (see the discussion of Sec. 5).

star.

(2) Case TS: we assumed that the stripped donor star loses all its initial information. When the star become smaller in mass, it follows the time scale of the main sequence star of that reduced mass.

We believe the realistic situation to lie between case TO and TS. The radius of the donor star, which essentially determines the orbital separation, is smaller in case TS because the donor star is less evolved than that in TO case. Even though, for any given donor star mass $M_{d}$, the lifetime of the donor star itself is longer in TS case, the smaller radius of the donor star gives the shorter orbital separation with larger mass loss rates, finally decreasing the lifetime of X-ray transients in case TS.

In Fig. 3, the time scales of X-ray transients are $\sim 2 \times 10^{9}$ yrs for a $1.25 M_{\odot}$ donor star. The mass of the donor star (initially a $1.25 M_{\odot}$ star) drops quickly in the beginning with a high mass loss rate $>10^{-9} M_{\odot} \mathrm{yr}^{-1}$, which slows down afterwards. Any donor stars $>0.5 M_{\odot}$ show the similar behaviour. Hence, independently of initial donor masses, most of the time X-ray transients will appear as low-mass X-ray transients (i.e. with a donor star mass $<0.5 M_{\odot}$ ) having a mass-loss rate of $\sim 10^{-10} M_{\odot} \mathrm{yr}^{-1}$, which is consistent with the empirical rate of $1.3 \times 10^{-10} M_{\odot} \mathrm{yr}^{-1}$ found by Van Paradijs (1996). The dot- 
dashed lines on the right panel of Fig. 3 correspond to the critical mass loss rate for the steady X-ray sources (King et al. 1997),

$$
\dot{M}_{c r i t} \approx 2.86 \times 10^{-11} M_{B H}^{5 / 6} M_{d}^{-1 / 6} P_{h r s}^{4 / 3} M_{\odot} \mathrm{yr}^{-1}
$$

We interpret the different masses of the main sequence companion as resulting firstly from varying initial masses, and secondly from the star being observed at different times in its evolution.

\section{Population Synthesis}

We calculated the total number of expected X-ray transients evolving through case C mass transfer in the same way as Brown, Lee \& Bethe (1999). From the discussions in Sec. 2, for a ZAMS star of mass $20 M_{\odot}$, we see that only the interval of $235 R_{\odot}$ between $1370 R_{\odot}$ and $1605 R_{\odot}$ of $a(t=0)$ is available for case $\mathrm{C}$ mass transfer. It is this small fractional interval that allows us to obtain a narrow interval of values for $\lambda \alpha_{c e}$. This is a logarithmic interval of only $\ln (1605 / 1370)=0.16$ compared with our total logarithmic interval of $\ln \left(4 \times 10^{9} \mathrm{~km} / 4 \times 10^{6} \mathrm{~km}\right)=7$ (Brown, Lee, \& Bethe 1999) so that we have a fraction of only 0.023 (as compared with 0.11 in Brown, Lee, \& Bethe 1999).

Since the possible ZAMS range for case C mass transfer is very narrow near $20 M_{\odot}$, we somewhat arbitrarily choose a ZAMS mass interval of $19 M_{\odot}<$ $M<22 M_{\odot}$. This means (Bethe \& Brown 1998) that the fraction of binaries with primaries in this range (assuming an IMF, $P(m) \propto M^{-2.5}$ ) is: $(1.9)^{-3 / 2}-$ $(2.2)^{-3 / 2}=0.08$ which is smaller by a factor of 2.5 from that for the interval of $20-35 M_{\odot}$ chosen by Brown, Lee \& Bethe (1999).

From the discussion in Sec. 3 and 4, the upper limit of the mass of the donor star is sensitive to the efficiency parameter $\lambda \alpha_{c e}$. For $\lambda \alpha_{c e}=0.4$ this upper limit is $1.6 M_{\odot}$. Assuming a flat $q$ distribution, we get $\Delta q \approx 1 / 20$. From Sec. 5 , we take the average lifetime of X-ray transients as $2 \times 10^{9} \mathrm{yrs}$, a factor two higher than the assumed lifetime in Brown, Lee \& Bethe (1999).

By taking the supernova rate as $2 \times 10^{-2} \mathrm{yr}^{-1}$ per Galaxy, we have the number of X-ray transients in our Galaxy as

$$
2 \times 10^{-2} \mathrm{yr}^{-1} \times \frac{1}{20} \times \frac{1}{2} \times 0.08 \times 0.023 \times 2 \times 10^{9} \mathrm{yr} \approx 1840
$$

where a binarity of a $1 / 2$ is considered as in Brown, Lee \& Bethe (1999). The total numbers in the Galaxy of such systems is estimated to be between a few 
hundred and a few thousand (Ergma \& Fedorova 1998). J Our estimate is thus consistent with theirs.

\section{Black Hole X-ray Binaries with (sub)Giant Donors}

Transient black hole binaries with (sub)giant donors might be expected to have followed the same scenario, but with initially higher companion masses and larger separations after the common envelope, so that they start mass transfer only when the donor has evolved off the main sequence. However, the large space velocity of Nova Scorpii 1994, best explained with a large amount of mass loss $\left(5-10 M_{\odot}\right)$ during the explosion in which the black hole was formed (Nelemans et al. 1999), and the high mass of the black hole in V404 Cyg $\left(\sim 10 M_{\odot}\right)$ suggest that the helium cores of stars around $20 M_{\odot}$ may not be massive enough to explain these systems. Hence, the progenitor masses of the black hole may have been larger.

The progenitor of the black hole in these systems might have been a massive WNL Wolf-Rayet star, as in the evolution of Brown et al. (2000a) of Cyg $\mathrm{X}-1$. They find for stars with ZAMS mass $\geq 60 M_{\odot}$, by applying reduced wind mass-loss rates, helium core masses after the WR stage of $\sim 15 M_{\odot}$. It should be noted that for such rapid rotating massive helium stars, magnetic coupling between the black hole and the accretion disc may give rise to a hypernova explosion accompanying a gamma-ray burst (Brown et al. 2000b).

\section{Conclusion}

We evolve the low-mass black-hole X-ray binaries, which are identified as the observed soft X-ray transients, and show that these systems are formed via case $\mathrm{C}$ mass transfer following helium core burning phase of the black hole progenitor. Although this scenario requires a set of fine tuned conditions, we are able to produce a current Galactic population of $\sim 2000$ objects, in agreement with estimates based on observations. Combining the narrow interval in initial separations leading to case $\mathrm{C}$ mass transfer with the allowed narrow range of separations after the common envelope evolution, we put constraints on the common envelope efficiency as $\lambda \alpha_{c e} \approx 0.2-0.5$.

5 Of the Wijers (1996) lower limit of 3000 transient black hole sources, 6 out of 9 sources had a main sequence companion with a short period, so he would have had $\sim 2000$ of the latter. 
Since our analysis requires fine tuned parameter space, more detailed calculations of the stellar evolution of stars with ZAMS masses around $20 M_{\odot}$ are required. More uncertain is the common envelope efficiency, which is essential for the formation of final short orbital period of the binaries.

\section{Acknowledgments}

This work was initiated by Gijs Nelemans' visit to Stony Brook. We acknowledge his helpful comments and discussions. GEB \& CHL were supported by the U.S. Department of Energy under grant DE-FG02-88ER40388.

\section{References}

[1] Brown, G.E., Lee, C.-H., and Bethe, H.A. 1999, New Astronomy 4, 313.

[2] Brown, G.E., Heger, A., Langer, N., Lee, C.-H., Wellstein, S., and Bethe, H.A. 2000a, in preparation.

[3] Brown, G.E., C.-H. Lee, Wijers, R.A.M.J., Lee, H.K., Israelian, G., and Bethe, H.A. 2000b, New Astronomy, 5, 191.

[4] Brown, G.E., Weingartner, J.C. and Wijers, R.A.M.J. 1996, ApJ, 463, 297.

[5] De Kool, M., Van den Heuvel, E.P.J., and Pylyser, E. 1987, A\&A, 183, 47.

[6] Dewi, J.D.M. and Tauris, T.M. 2000, A\&A, 360, 1043.

[7] Ergma, E. and Fedorova, A. 1998, A\&A, 338, 69.

[8] Kalogera, V. 1999, ApJ, 521, 723.

[9] Kalogera, V. and Webbink, R.F. 1998, ApJ, 493, 351.

[10] King, A.R., Kolb, U., and Szuszkiewicz, E. 1997, ApJ, 488, 89.

[11] Nelemans, G., Tauris, T.M., and van den Heuvel, E.P.J. 1999, A\&A, 352, L87.

[12] Paczyński, B. 1976, in P.P. Eggleton, S. Mitton, J. Whelan (eds.), Structure and evolution of close binary systems, p. 75, Kluwer, Dordrecht.

[13] Portegies Zwart, S., Verbunt, F., and Ergma, E. 1997, A\&A, 321, 207.

[14] Rauw, G., Vreux, J.-M., Gosset, E., Hutsemékers, D., Magain, P., and Rochowicz, K. 1996, A\&A, 306, 771.

[15] Schaller, G., Schaerer, D., Meynet, G., and Maeder A. 1992, A\&AS, 96, 269.

[16] Skumanich, A 1972, ApJ, 171, 565. 
[17] Stepien, K. 1995, MNRAS, 274, 1019.

[18] Tauris, T.M., and Dewi, J.D.M., 2001 A\&A in press.

[19] Van Paradijs, J. 1996, ApJ, 464, L139.

[20] Verbunt, F., in: Neutron Stars and Their Birth Events. Ed. W. Kundt, Kluwer, Dordrecht p.179

[21] Wellstein, S., and Langer, N. 1999, A\&A, 350, 148.

[22] Wijers, R.A.M.J. 1996, Evolutionary Processes in Binary Stars, Vol. 327, edited by Wijers et al., Kluwer Acad. Publ., Dordrecht.

[23] Woosley, S.E., and Weaver, T.A. 1995, ApJS, 101, 181. 